\title{
Challenges associated with Quartz Crystal Microbalance with Dissipation (QCM-D) as a wettability screening tool
}

\author{
Samuel Erzuah ${ }^{1,2, *}$, Ingebret Fjelde ${ }^{1,2,3}$, and Aruoture Voke Omekeh ${ }^{1,3}$ \\ ${ }^{1}$ The National IOR Centre of Norway, P.O. Box 8600 Forus, N-4036 Stavanger, Norway \\ 2 The University of Stavanger, P.O. Box 8600 Forus, N-4036 Stavanger, Norway \\ ${ }^{3}$ International Research Institute of Stavanger (IRIS), P.O. Box 8046, N-4068 Stavanger, Norway
}

Received: 28 February 2018 / Accepted: 21 September 2018

\begin{abstract}
Wettability is an indispensable parameter in multiphase flow due to its profound effect in fluid phase distribution and flow properties in the oil reservoirs. One approach of unravelling the enigma associated with wettability characterization is to investigate oil adhesion onto reservoir rock surface during crude oil accumulation. This was accomplished using Quartz Crystal Microbalance with Dissipation (QCM-D) device. The QCM-D is a microbalance device that hinges on the changes in the frequency of a resonating crystal due to changes in the mass on sensor surface, precipitation, adsorption and desorption. However, this technique was confronted with numerous challenges during its early try-out. The objective of this study is to enumerate these challenges and how they were resolved. The piston-cell, valves, flow-lines and most of the experimental set-up were made from stainless steel. Hence, the high temperature coupled with high salinity brine resulted in the formation and deposition of corroded materials on the sensor. Due to the high sensitivity of the QCM-D technique, these corrosion deposits were detected via the high attenuation of the frequency signal as time elapsed during Formation Water (FW) injection. The second challenge was related to the dissolution of the thin sensor coatings (sensor etching) depicted by the relatively high increase in frequency signal with negligible changes in Dissipation (D). The third challenge was related to the trapping of fluids such as Stock Tank Oil (STO) inside the flow-cell. Finally, salt precipitation resulting from temperature variation during the initial experimental set-up was also observed. To resolve the corrosion challenge, all the stainless-steel components in the experimental set-up were replaced with titanium and non-metallic component such as peek materials. The sensor etching was also averted by injecting the brine through a packed column filled with similar mineral as the coatings on the sensor to attain equilibrium prior to injecting it onto the sensor. Geochemical simulation of the sensor etching was also confirmed using the geochemical simulator PHREEQ-C. Furthermore, the trapping of fluids inside the flow-cell was overcome by rotating the flow-cell to optimize the fluid displacement via capitalizing on their density contrast. Finally, the salt precipitation was avoided by conducting the experiment in a constant temperature experimental set-up. The QCM-D technique can be employed to estimate wettability by evaluating the tendency of the various minerals to adhere oil. The beauty of the QCM-D technique is that the surface interactions can be monitored on a real-time.
\end{abstract}

\section{Symbols and nomenclature}

$\begin{array}{ll}\text { BPV } & \text { Back-Pressure Valve } \\ \text { COBR } & \text { Crude Oil/Brine/Rock } \\ \text { DW } & \text { Distilled Water } \\ \text { FW } & \text { Formation Water } \\ \text { LSW } & \text { Low Salinity Water } \\ \text { QCM-D } & \text { Quartz Crystal Microbalance with Dissipation } \\ \text { STO } & \text { Stock Tank Oil }\end{array}$

* Corresponding author: samuel.erzuah@iris.no

\author{
$\Delta D \quad$ Change in Dissipation (ppm) \\ $\Delta f \quad$ Change in frequency $(\mathrm{Hz})$ \\ QHPT High-Pressure High-Temperature QCM-D
}

\section{Introduction}

The QCM-D is a highly sensitive weight detection and monitoring device that has permeated the fiber of modern industries such as the pharmaceutical, biomedical, oil and gas 
industry. The QCM-D relies on the changes in the resonance frequency of the oscillating crystal to estimate the mass of the adsorbed film (Alagha et al., 2013; Chandrasekaran et al., 2013; Ekholm et al., 2002; Feiler et al., 2007; Keller and Kasemo, 1998). This is achieved by measuring the changes in the frequency $(\Delta f)$ and dissipation $(\Delta D)$ with time. Aside the effect of the adsorbed film on the frequency and dissipation signal, the properties of the injected fluids such as density and viscosity also influence the signals. For instance, bulk shift in the frequency and dissipation signals occurs during the injection of two immiscible fluids of varying properties such as Formation Water (FW) and $n$-decane consecutively. The bulk shift in the frequency and dissipation signals has no effect in the adsorption process. Considering $\mathrm{FW} / n$-decane/FW injection sequence, the constant FW frequency before and after injecting $n$-decane was reported by Erzuah et al. (2018) to depict lack of oil adsorption. On the other hand, adsorption/desorption onto the sensor is also depicted by a gradual decrease/increase in the frequency signal due to the added mass unlike the bulk shift in the frequency and dissipation signal resulting from the fluid properties. Erzuah et al. (2018) reported that the frequency signals of the oscillating crystal with adsorbed film is lower than prior to the adsorption during the injection sequence. The dissipation signal $(\Delta D)$ is proportional to the magnitude of the adsorbed mass. The frequency signal $(\Delta f)$ on the other hands is inversely proportional to the adsorbed mass.

Numerous researchers have carried out comprehensive protein adsorption studies using QCM-D due to its role in the successful implantation of prosthetic devices in the human body (Bluemmel et al., 2007; Feiler et al., 2007). Cao et al. (2018) also investigated calcite growth rate using QCM-D. Nguyen and Elimelech (2007) have carried out comprehensive studies on the adsorption of plasmid DNA via QCM-D. Adsorption of cell onto various surfaces have also been carried out (Dixon, 2008). Cattanach et al. (2011) undertook corrosion monitoring under sales gas condition using QCM-D.

The intended application of the QCM-D in this presented study was to investigate oil adhesion onto reservoir rock surface. However, this technique was confronted with numerous challenges during the early try-out. The aim of this study is to enumerate these challenges and how they were averted in a chronological manner. Since the Stock Tank Oil (STO) contained volatile components, a QCM-D cell that allows higher pressure was used to avoid boiling.

\section{Overview of the HPHT QCM-D device (QHPT)}

The aim of this presented study has always been to qualify the QCM-D device as a wettability characterization tool. To accomplish this, the High-Pressure, High-Temperature (HPHT) QCM-D vessel (QHPT) developed by Biolin Scientific (Frölunda, Sweden) was employed in this study. The conventional QCM-D device can accommodate only one fluid in the flow-cell chamber at a given time. In other words, the active electrode area of the sensor is filled with the test fluid while the non-active part of the sensor is occupied by air. The QHPT vessel on the other hand can accommodate two fluids in the flow-cell chamber at a given time namely; the test and the system fluids. The test fluid occupies the active electrode area/deposition area of the sensor while the system fluid also fills the flow-cell chamber beneath the sensor (non-active part of the sensor). The system fluid used in this study was $n$-decane while the test fluids were crude oil and brine. The magnitude of the dissipation signal depends on the properties of the fluids occupying each side of the sensor. For instance, if air occupies both sides of the sensor as observed during the start of the experiment (Fig. 1A), the energy loss by the resonating sensor resulting from the presence of air above and beneath the sensor is negligible $(\sim 0 \mathrm{ppm})$. This is because, the properties of air such as density and viscosity are negligible. Hence, the contribution of air to the energy loss (absolute dissipation) is zero.

However, at the start of the experiment throughout this study, the system fluid used in the QHPT vessel (Fig. 1B) was $n$-decane while the test fluid (reference fluid) was FW. Both the $n$-decane and the FW have different properties (densities and viscosities) than air and hence, the energy loss by the resonating sensor due to the presence of these fluids will not be the same as in an air-filled flow-cell (Fig. 1A). In other words, the absolute dissipation value at the start of the experiment depends on the properties (density and viscosity) of fluids in the flow-cell chamber at the onset of the experiment. Nonetheless, the changes in frequency $(\Delta f)$ and dissipation $(\Delta D)$ signals were used throughout this study but not their absolute values.

In an attempt to qualify the QCM-D as wettability screening tool, the challenges that were confronted and how they were averted are presented in a chronological manner.

\section{Challenges}

These challenges include corrosion, sensor etching, fluid trapping and salt precipitation. These challenges are presented in a sequential order as they occurred during the qualification of the QCM-D technique as a wettability screening tool.

\subsection{Corrosion}

The corrosion challenge is presented first followed by its mitigation protocol.

\subsubsection{Corrosion challenge}

The QCM-D technique is a highly sensitive weight measuring device. Hence, it has the capacity of detecting contaminants originating from the experimental set-up such as corrosion deposit. Considering Part I of Figure 2, it can be observed that the frequency and the dissipation signals were relatively stable during the injection of Distilled Water (DW), i.e. no change in mass on sensor. However, gradual attenuation of the frequency signal was observed during Formation Water (FW) injection Part II of Figure 2 with 

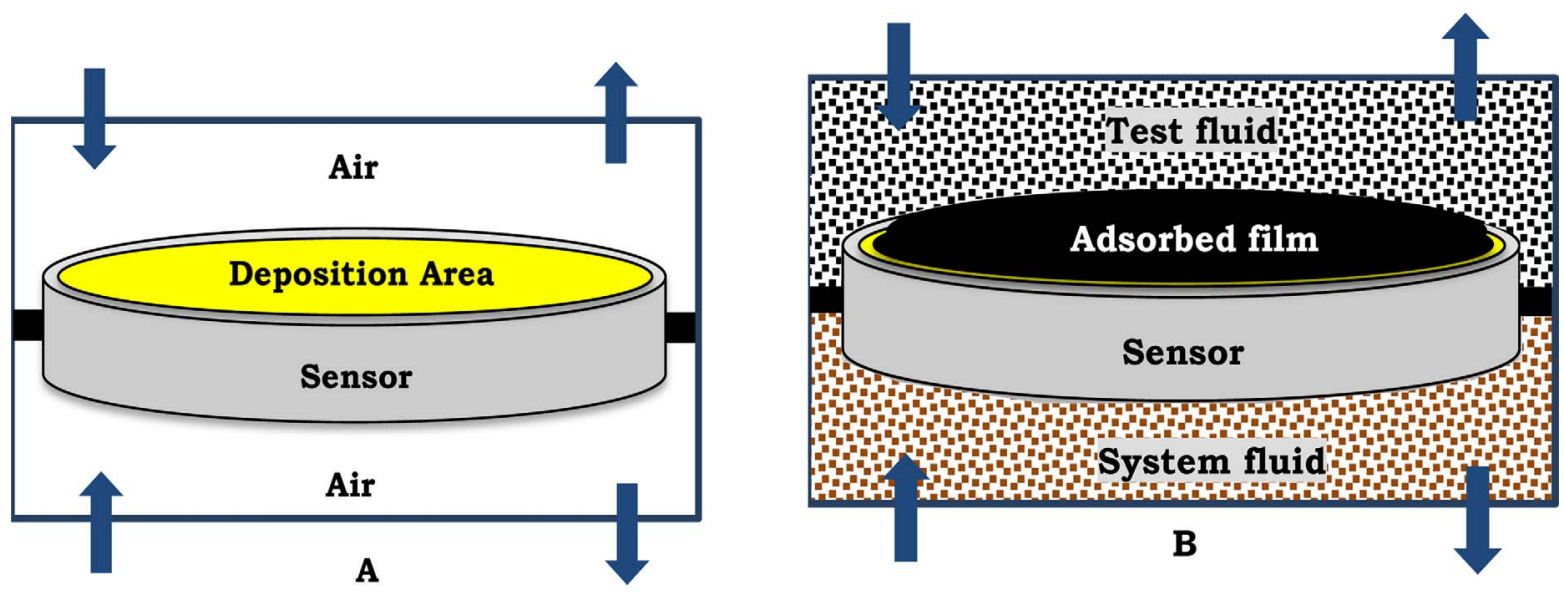

Fig. 1. Analogy of the fluids on both the active (deposition area) and non-active electrodes part of the high-pressure hightemperature QCM-D (QHPT) vessel. If air occupies both sides of the sensor (A), the energy loss (Dissipation energy) by the sensor is negligible $(\sim 0)$. On the other hand, if the system fluid occupies the non-active part of the sensor whiles the test fluid also occupies the active electrode part of the sensor (B). Hence, the total energy lost by the sensor is due to the presence of the fluids above and beneath the sensor in addition to the attenuation due to the adsorbed film.

a corresponding increase in the dissipation energy. Hence, it can be inferred that the additional mass due to the corrosion deposit is the reason for the observed trend (Fig. 2).

As compared to the DW signal, the attenuation of the frequency signal cannot be attributed to the presence of ion in the FW alone but rather its interactions with its environment. This was depicted by the gradual attenuation of the frequency signal describing deposition. When the flowcell was opened, it was observed that the reduction in the frequency signal with time during the FW injection was due to the formation and/or deposition of corroded material onto the sensor. The corrosion deposit was due to the high salinity brine with $\mathrm{pH}$ of 5.9 coupled with high temperature in the presence of corrosive materials such as stainless steel in experimental set-up. Thus, providing a conducive condition for corrosion to take place (Fig. 2).

To confirm this hypothesis, a stainless-steel piston-cell was filled with the FW for barely a week. It can be observed from Figure 3 that a yellowish-brown substance was formed on the walls of the stainless-steel piston-cell in addition to changes in colour of the FW from colourless (Fig. 5A) to yellow (Fig. 5B). Thus, confirming that corrosion has taken place as supported by Figure 2 .

\subsubsection{Mitigation of the corrosion challenge}

To resolve the corrosion challenge, a similar test was performed by storing the FW in titanium piston-cell for barely a week. It can be observed from Figure 4 that; no colour change was observed on the wall of the titanium piston-cell unlike in the stainless-steel (Fig. 3). Contrary to the change in colour of the FW stored in the stainless-steel piston-cell, the FW remained colourless after storing it in the titanium piston-cell (Fig. 5C). This confirms that no corrosion has taken place. Hence, the flow-cell and all the stainless-steel components in the experimental set-up were replaced with titanium and non-metallic component such as peek materials.

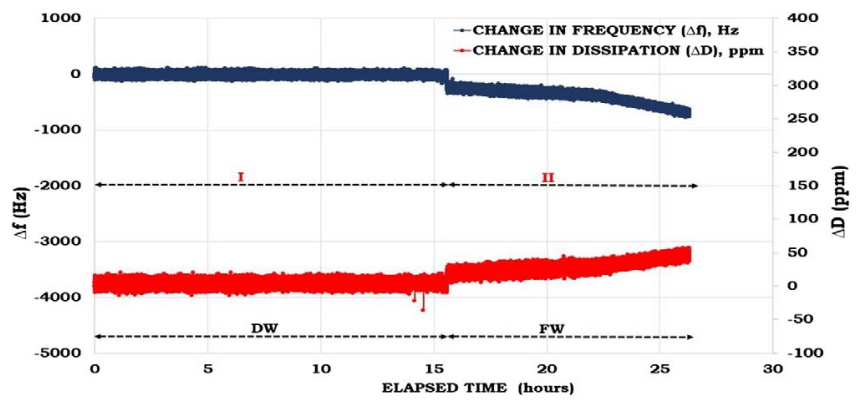

Fig. 2. Corrosion deposits originating from the experimental set-up, high temperature and the high salinity brine.

In addition, Inductive Coupled Plasma (ICP) analytical technique was employed to examine the composition of the original FW (Fig. 5A) and the FW stored in both the stainless-steel piston-cell (Fig. 5B) and that of the titanium piston cell (Fig. 5C). For the FW stored in the stainless-steel piston cell (Figs. 3 and 5B), precipitation of corrosion deposits was observed as time elapse. Hence, the sample in Figure 5B was separated into two halves. Half of this separated volume was analysed without adding hydrochloric acid $(\mathrm{HCl})$ while $0.1 \%$ volume of $\mathrm{HCl}$ was added to the other half to dissolve the dispersed precipitate. It can be observed from Table 1 that the concentration of $\mathrm{Fe}$ in both the original $\mathrm{FW}$ and the $\mathrm{FW}$ stored in the titanium piston-cell was less than $0.1 \mathrm{mg} / \mathrm{L}(<0.1 \mathrm{mg} / \mathrm{L})$. This confirms that the corrosion challenge can be mitigated by using the titanium piston-cell (Fig. 4) as observed in Figure 5C. On the other hand, the content of $\mathrm{Fe}$ in the $\mathrm{FW}$ stored in the stainlesssteel piston cell was greater than $0.1 \mathrm{mg} / \mathrm{L}(>0.1 \mathrm{mg} / \mathrm{L})$, thus confirming that corrosion has taken place.

In addition, it can also be observed that the concentration of Fe in the FW stored in the stainless-steel piston-cell (Fig. 5B) without adding $\mathrm{HCl}$ was relatively small $(0.1 \mathrm{mg} / \mathrm{L})$ as compared to that with $\mathrm{HCl}$ added 


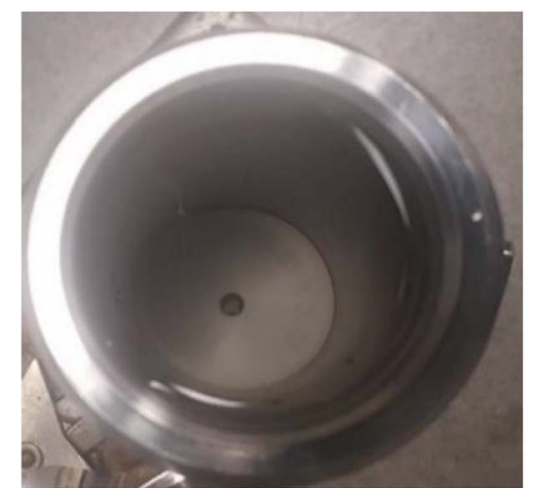

A

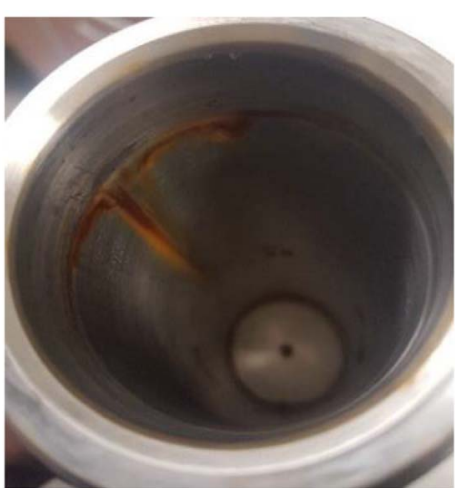

B

Fig. 3. Change in colour of the stainless-steel piston-cell wall before (A) and after (B) storing FW in it for 7 days. The yellowishbrown substance formed at the walls of the stainless-steel piston-cell can be linked to the corrosion formation.

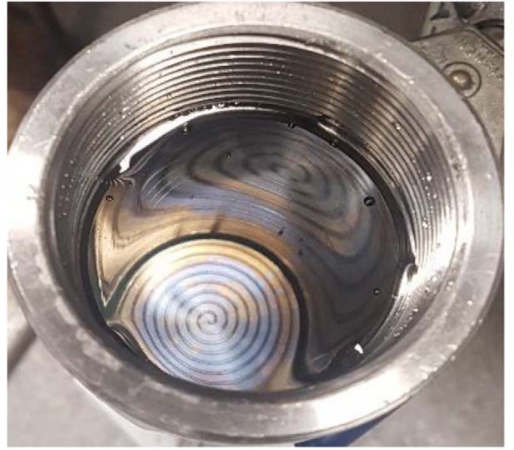

A

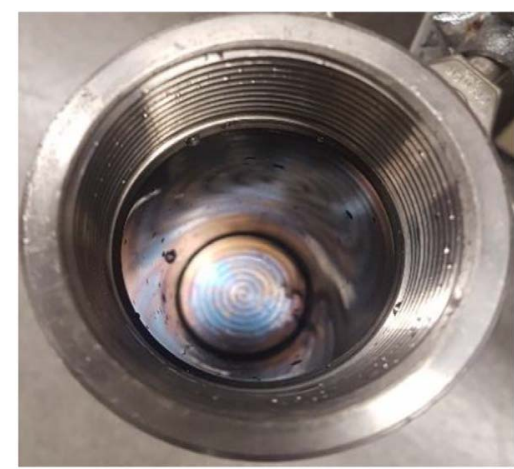

B

Fig. 4. Illustrate no corrosion resulting from the negligible change in colour of the wall of the titanium piston-cell before (A) and after (B) storing the FW in it for 7 days. Thus, confirming that titanium component and non-metallic component can mitigate the corrosion challenge.

$(8.2 \mathrm{mg} / \mathrm{L})$. The increase in the Fe content in the latter FW was attributed to the dissolution of the precipitated corrosion deposit with the $\mathrm{HCl}$. To add to the above, it can be concluded that titanium piston-cell did not react with the FW as confirmed by the negligible titanium content $(<0.04 \mathrm{mg} / \mathrm{L})$ in all the studied FW conditions. Hence, the corrosion challenge can be mitigated by the titanium piston-cell and non-metallic components such as Polyether Ether Ketone (PEEK).

\subsubsection{Experiment with resolved corrosion challenge}

From Part I of Figure 6 it can be observed that no corrosion took place due to the relatively stable frequency and dissipation signal during FW injection unlike in Figure 2 with stainless-steel components. However, there was an increase in frequency of the signal with time (Part II of Fig. 6).

This was attributed to the dissolution of the coatings on the sensor with time leading to high oscillation of the resonating crystal. This challenge is termed "sensor etching". It can be observed from Part III of Figure 6 that the frequency and dissipation signals were lost as time elapsed.

\section{Sensor etching}

Like the presentation of the corrosion challenge, the sensor etching challenge will be presented first followed by its mitigation.

\subsection{Sensor etching challenge}

The QCM-D sensor is composed of quartz crystal with a thin layer $(50-100 \mathrm{~nm})$ of the desired mineral such as quartz and kaolinite. During the injection of high salinity brine, the coatings on the sensors were dissolved. This was depicted by the relatively high frequency signal without any significant changes in Dissipation (D). In other word, etched sensors led to reduction in mass of the crystal thereby increasing the frequency without any appreciable changes in the dissipation signal unlike during adsorption/deposition (Fig. 2). Figure 7 shows the nature of a quartz sensor before and after etching. Figures 6 and 8 also illustrate the etching of quartz and kaolinite sensors respectively during $\mathrm{FW}$ injection.

Unlike the quartz sensor (Fig. 6), the etching of the kaolinite sensor (Fig. 8) was very fast. In other words, the kaolinite sensor begun to etch approximately $4 \mathrm{~h}$ after 


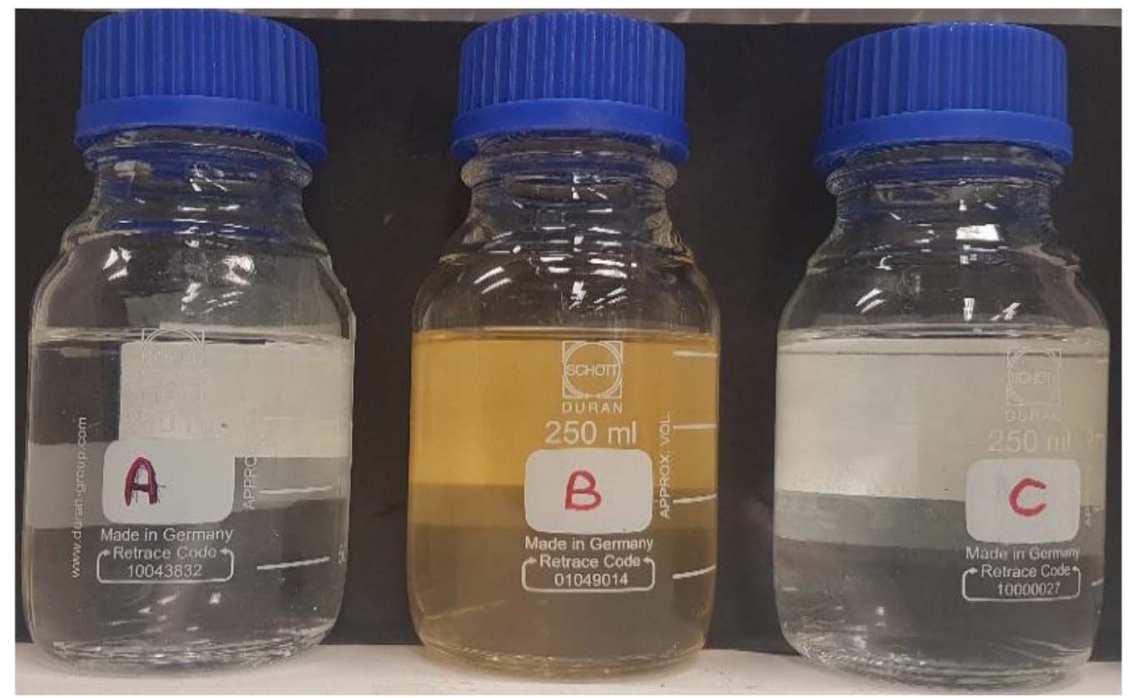

Fig. 5. Change in colour of the prepared FW (A) when stored in stainless steel (B) and titanium (C) piston cells respectively. The colour change in FW (B) can be attributed to corrosion formation resulting from the stainless-steel piston-cell coupled with the corrosive nature of the studied system. Since the prepared FW and the brine store in titanium piston-cell were both colourless (A and $\mathrm{C}$ respectively). It confirms that the corrosion challenge can be mitigated by using titanium piston-cell.

Table 1. Composition of the FW.

\begin{tabular}{|c|c|c|c|c|}
\hline$\underline{\text { Element }}$ & Original FW mg/L & $\begin{array}{l}\text { FW-stainless steel } \\
\text { (without } \mathrm{HCl} \text { ) } \mathrm{mg} / \mathrm{L}\end{array}$ & $\begin{array}{l}\text { FW-stainless steel } \\
\text { (with } \mathrm{HCl} \text { ) } \mathrm{mg} / \mathrm{L}\end{array}$ & FW-Titanium mg/L \\
\hline Sodium (Na) & 30600 & 30800 & 30100 & 30500 \\
\hline Calcium (Ca) & 5920 & 6010 & 5970 & 6000 \\
\hline Magnesium (Mg) & 419 & 422 & 415 & 415 \\
\hline Barium (Ba) & 0.23 & 0.21 & 0.29 & 0.20 \\
\hline Iron $(\mathrm{Fe})$ & $<0.1$ & 0.1 & 8.2 & $<0.1$ \\
\hline Strontium (Sr) & 3.25 & 2.99 & 3.09 & 3.05 \\
\hline Potassium (K) & 245 & 247 & 243 & 246 \\
\hline Titanium (Ti) & $<0.04$ & $<0.04$ & $<0.04$ & $<0.04$ \\
\hline Sulfur (S) & 31.4 & 31.4 & 30.9 & 31.1 \\
\hline
\end{tabular}

the inception of the experiment (Part I of Fig. 8) as opposed to approximately $10 \mathrm{~h}$ in the case of the quartz sensor (Part I of Fig. 6). This confirms that the dissolution rate of kaolinite is high compared to quartz.

\subsection{Mitigation of the sensor etching challenge}

The sensor etching challenge was averted by injecting the brine through a pre-column filled with a similar material as the sensor coating. This was to attain equilibrium between the mineral and $\mathrm{FW}$ before it reaches the sensor. From Part I of Figure 9, it can be observed that no etching took place during the injection of DW. However, the sensor begun to etch during the injection of $\mathrm{FW}$ without packed column (Part II of Fig. 9). However, when the FW was injected through a packed column to attain equilibrium with the coatings on the sensor, etching was averted (Part III of Fig. 9).

A completely packed sand column poses no threat to the flow of brine due to its high permeability. On the contrary, a completely packed kaolinite column will hinder the flow of brine due to its low permeability. There was therefore the need to assess a kaolinite-quartz mixture that can mitigate both etching and permit the flow of the injected brine.

Three (3) different kaolinite-quartz mixtures were considered to evaluate their effect on sensor etching. It can be concluded from Part I of Figure 10 that, if the amount of kaolinite in the mixture is small, it will lead to etching. This is because, it will take a longer time for the brine to attain equilibrium with the minerals in the pre-column. This is confirmed by Parts II and III of Figure 10 with 


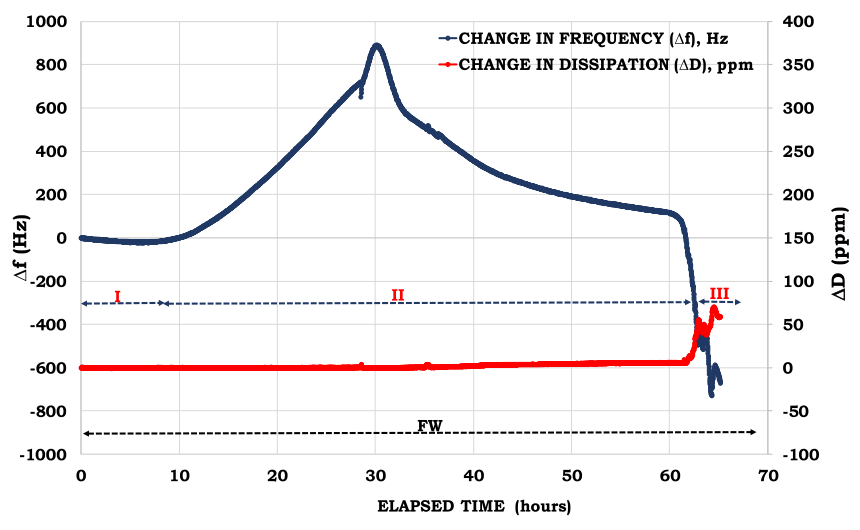

Fig. 6. QCM-D experiment with resolved corrosion challenge with using quartz sensor.

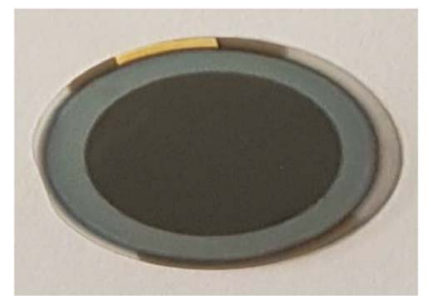

A

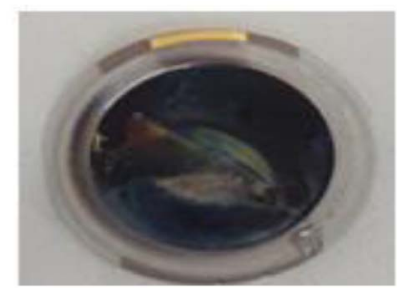

B
Fig. 7. Quartz sensor surface before (A) and after (B) etching.

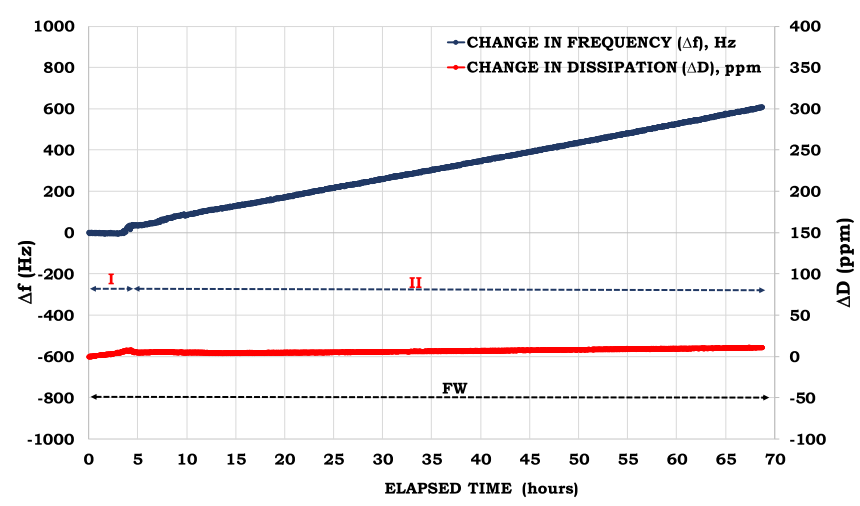

Fig. 8. Etching of kaolinite sensor due to high salinity brine coupled with high temperature $\left(65^{\circ} \mathrm{C}\right)$.

relatively stable frequency signal resulting from the shorter period required to reach equilibrium due to the relatively high amount of kaolinite in the mixture as compared to Part I. In addition, it can be concluded that Part II was more stable than Part III due to the relatively high amount of kaolinite in the kaolinite-quartz mixture in the former than the latter. Hence, the optimal kaolinite-quartz mixture that can mitigate etching and promoted brine injection was that of Part II of Figure 10. The sensor etching challenge and its mitigation was also confirmed by a geochemical simulator, PHREEQ-C. The geochemical simulation results confirm the dissolution of the sensor the coatings without pre-column (Fig. 11). From Figures 10 and 11, it can be

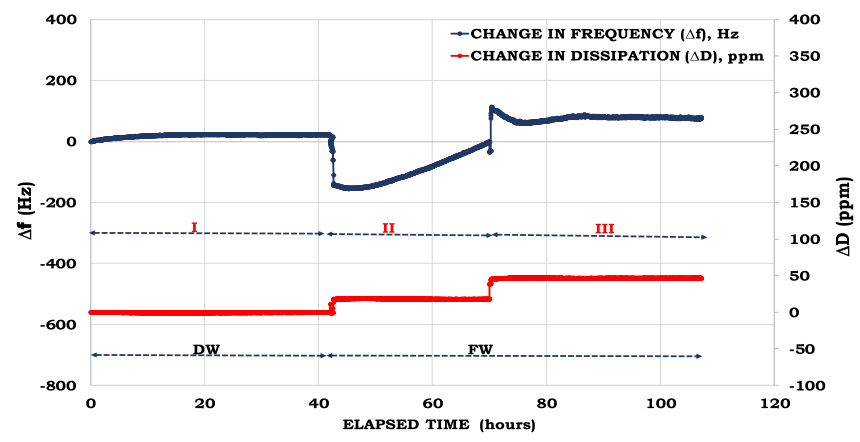

Fig. 9. Illustrates sensor etching mitigation with packed column (sand packed). Part II without and Part III with precolumn.

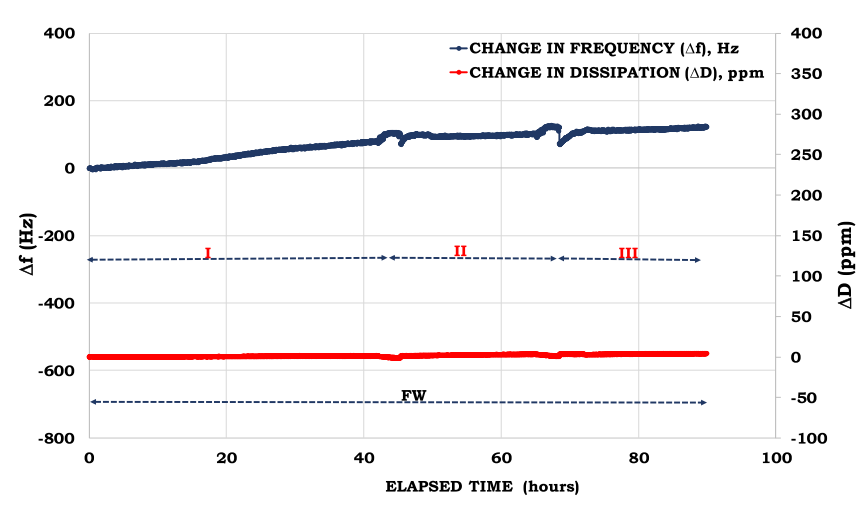

Fig. 10. Evaluating the quartz-kaolinite mixture that can curb etching with high permeability.

concluded that pre-column is needed if sensor etching is to be averted.

\subsection{Experimental result with resolved sensor etching challenge}

From Figure 12, it can be observed that the FW frequency signal looks relatively stable before and after the injecting $n$ decane. However, the bulk shift in the frequency signal during the injection of $n$-decane (Part II of Fig. 12) after the injection of $\mathrm{FW}$ is as a result of the difference in fluid properties.

Nonetheless, the bulk shift has no effect on the adsorption process. The LSW frequency and dissipation signals also look relatively stable. Thus, confirming that the sensor etching has been averted.

\section{Trapping of fluids}

The fluid trapping challenge was presented first followed by how it was mitigated.

\subsection{Challenge with trapping of fluids inside the flow-cell}

During the $\mathrm{FW} / N$-Decane/FW $/ \mathrm{LSW} / \mathrm{FW}$ injection sequence (Fig. 13), the effect of the trapped fluids such as 

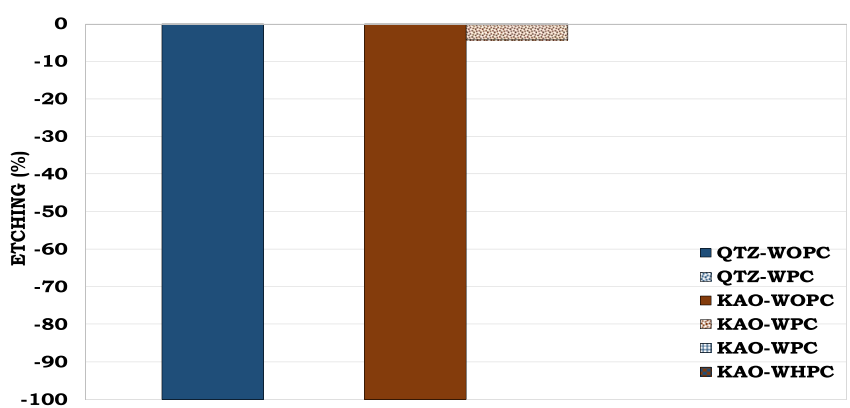

Fig. 11. Geochemical simulation of the sensor etching via PHREEQ-C. WOPC $=$ Without pre-column, $\mathrm{WPC}=$ With pre-column, WHPC $=$ With hybrid pre-column, $\mathrm{QTZ}=$ quartz sensor and $\mathrm{KAO}=$ kaolinite sensor.

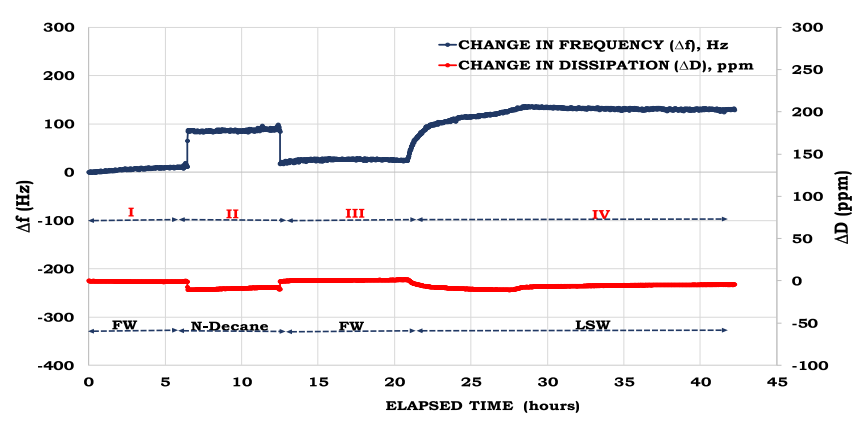

Fig. 12. $\mathrm{FW} / \mathrm{N}$-Decane/FW injection sequence to evaluate the mitigation of the sensor etching phenomenon.

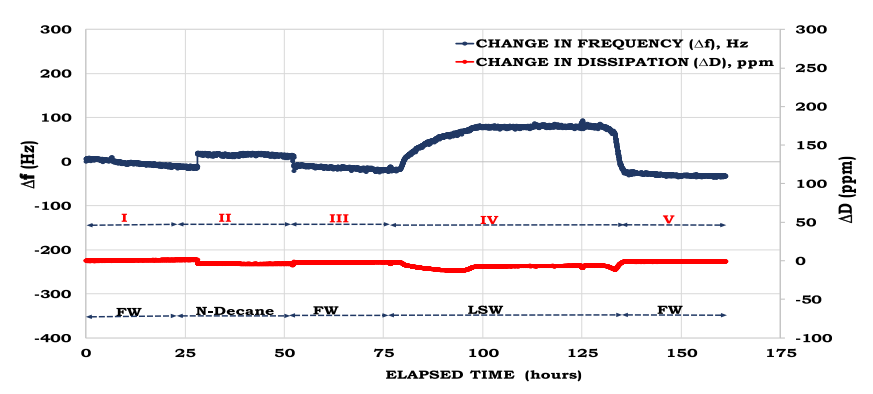

Fig. 13. Trapping of fluid inside the flow-cell due to density contrast.

the oil was not distinct due to lack of polar components in the $n$-decane. However, during the $\mathrm{FW} / \mathrm{STO} / \mathrm{FW} / \mathrm{LSW} /$ FW injection sequence (Fig. 14 and Fig. 15), it was observed that the frequency of the FW signal after injecting STO (Part III of Fig. 14) was decreasing with time depicting adsorption as observed during the STO injection (Part II of Fig. 14).

However, the LSW frequency signal was relatively stable (Fig. 14). This can be attributed to the low concentration of divalent cations available to bridge the trapped oil during LSW injection (Part IV of Fig. 14) as compared to the FW injection (Part III of Fig. 14).

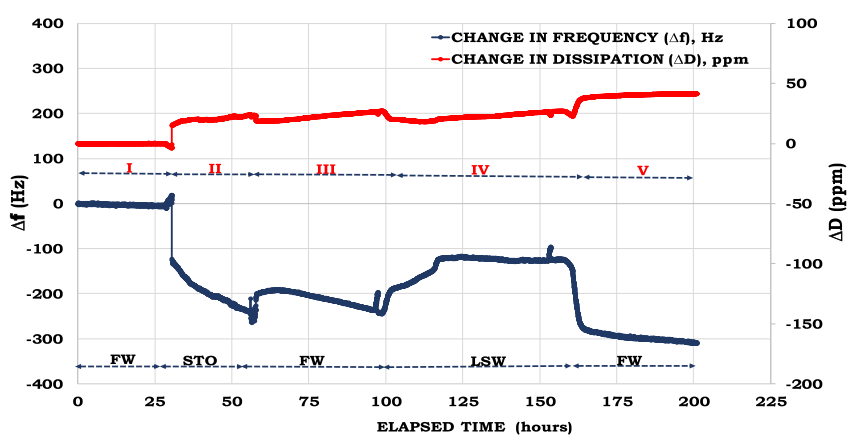

Fig. 14. Trapping of fluid inside the flow-cell due to density contrast during $\mathrm{FW} / \mathrm{STO} / \mathrm{FW} / \mathrm{LSW} / \mathrm{FW}$ injection sequence.

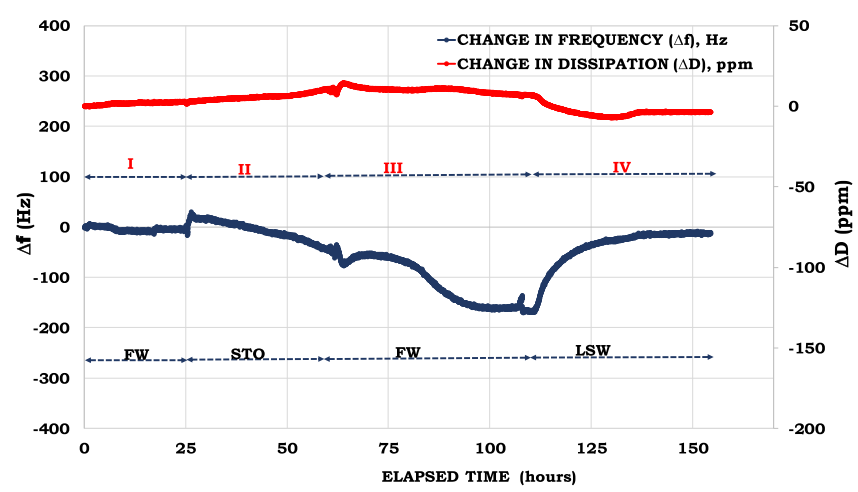

Fig. 15. Trapping of fluid inside the flow-cell due to density contrast during $\mathrm{FW} / \mathrm{STO} / \mathrm{FW} / \mathrm{LSW} \# 1$ injection sequence.

This was depicted by the attenuation of the frequency signal during the injection of the FW after the STO injection (Part III of Fig. 14). From Figure 15, it can be observed that most of the trapped STO were displaced after a prolonged injection period. This resulted in a stable frequency signal during FW injection (Part III of Fig. 15) unlike in Part III of Figure 14. In other words, it took longer time to displace most of the trapped oil from the flow-cell before the FW frequency signal became stable (Part III of Fig. 15).

\subsection{Mitigation of the challenge with trapped oil inside the flow-cell}

Mitigation of the trapped fluids inside the flow-cell was achieved by rotating the flow-cell to optimize the displacement of the previously injected fluid with the current one by capitalizing on their density contrast. This resulted in a fast stabilization time during the injection of $\mathrm{FW}$ after injecting STO. During the displacement of the FW with the oil, the flow-cell was rotated such that maximum contact was established between the sensor and the oil due to density contrast. In other words, the flow-cell was rotated in such a way that the less dense fluid rises to the top to occupy the surface of the sensor while the denser fluid moves to the bottom of the flow-cell chamber to be expelled (Fig. 16).

From Figure 17, it can be observed that during the displacement of the oil with the FW, the flow-cell was 


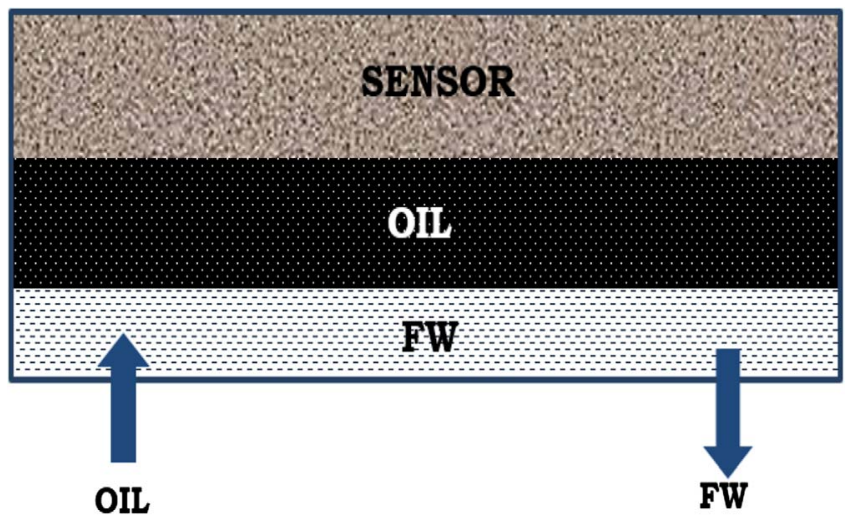

Fig. 16. Displacement of the denser fluid (FW) with the less dense fluid (oil).

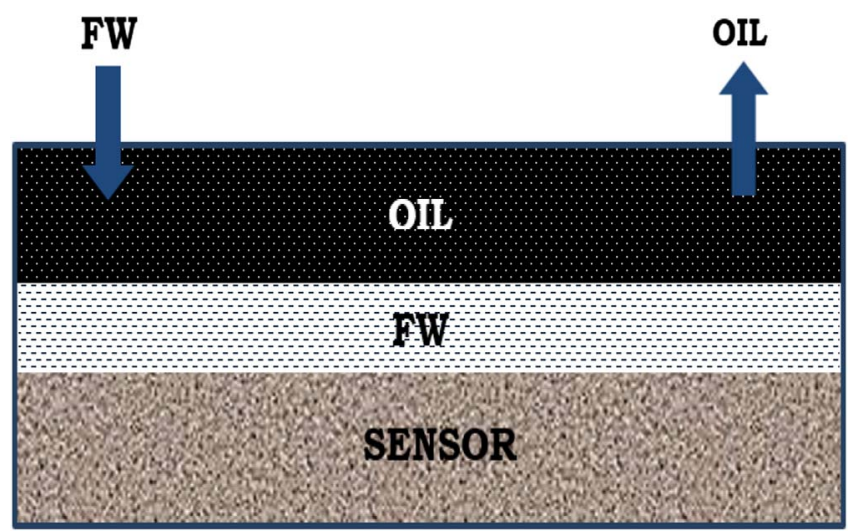

Fig. 17. Displacement of the less dense fluid (oil) with the denser fluid (FW).

rotated such that the denser fluid (FW) sink to the bottom of the flow-cell chamber to cover the sensors surface. The less dense fluid on the other hand rises to the top of the flow-cell chamber to be displaced due to density contrast.

\section{Temperature}

\subsection{Salt precipitation challenge and its mitigation}

Finally, there was also the tendency for salt precipitation resulting from temperature variation in the initial experimental set-up. This was mitigated by conducting the experiment in a constant temperature environment as depicted in Figure 18.

\section{Developed QCM-D method}

\subsection{Experimental procedure and set-up}

The QCM-D experimental procedure can be grouped into three (3) main categories namely; preparatory

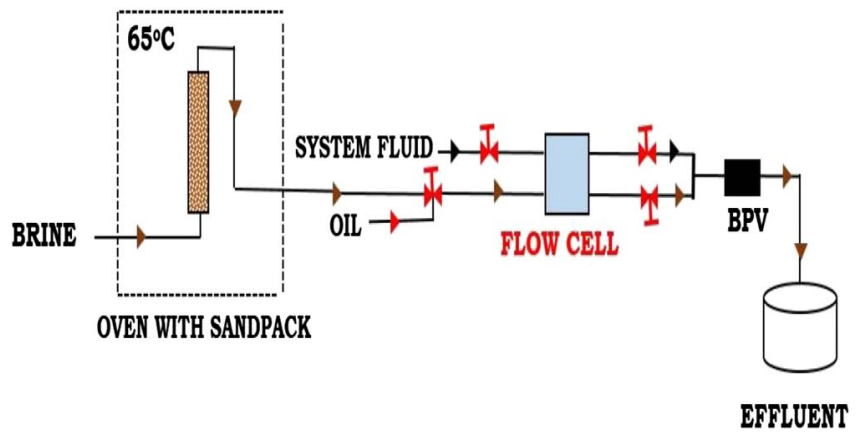

Fig. 18. Experimental set-up with QCM-D challenges resolved $(\mathrm{BPV}=$ Back Pressure Valve).

(pre-acquisition) stage, acquisition stage and postacquisition stage. A schematic illustration of the QCM-D experimental set-up is shown in Figure 18.

\subsubsection{Preparatory stage (pre-acquisition)}

The flow-cell and the flow-lines were thoroughly cleaned with ethanol and dried with nitrogen gas to remove impurities from the system before mounting the sensor. The sensor was thoroughly cleaned with ethanol and dried with nitrogen gas before mounted into the flow cell. The temperature of the flow-cell was then set to the desired reservoir temperature $\left(65^{\circ} \mathrm{C}\right.$ used in this study). The air in the system was displaced with ethanol using high and low flow rate intermittently. The ethanol in the non-active part of the flow-cell was displaced with the system fluid ( $n$-decane) while that at the active electrode was displaced with the desired brine. Back Pressure Valve (BPV) is required at the outlet as illustrated in Figure 18 to avoid boiling of oils. The tubings from the active and the non-active parts of the sensor are connected to ensure that the pressure is the same on both sides of the sensor. This is to prevent losing the sensor through cracking.

\subsubsection{Acquisition stage}

The log was restarted after a stable baseline was achieved. The desired injection fluids were injected in their desired order while monitoring the frequency and the dissipation signals on real-time. When switching between fluids, the pump should be stopped with all the valves closed without stopping the data acquisition. Prior to injecting the next fluid, it should be ensured that pressure of the injecting fluid was approximately the same as in the flow-cell.

\subsubsection{Post-acquisition stage}

At the end of the acquisition period, the pump was stopped, and the acquisition was stopped. The BPV was then gradually disconnected to bleed off the pressure in the flow-cell. The sensor was then removed from the flow-cell and kept for latter analysis. A dummy sensor was then mounted in the flow-cell prior to injecting the cleaning fluid. The designated cleaning fluid for $n$-decane is ethanol while that of the STO is toluene followed by ethanol. 


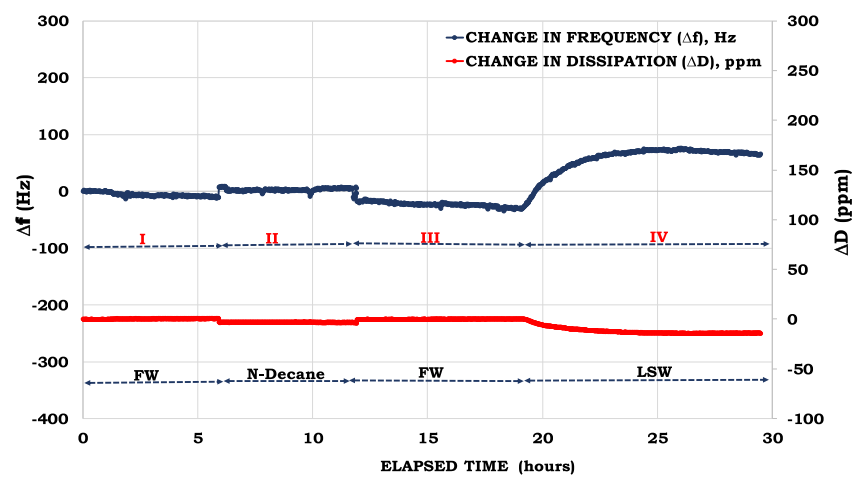

Fig. 19. Frequency and dissipation signals during FW/ $\mathrm{N}$-Decane/FW/LSW\#1 injection sequence using quartz sensor.

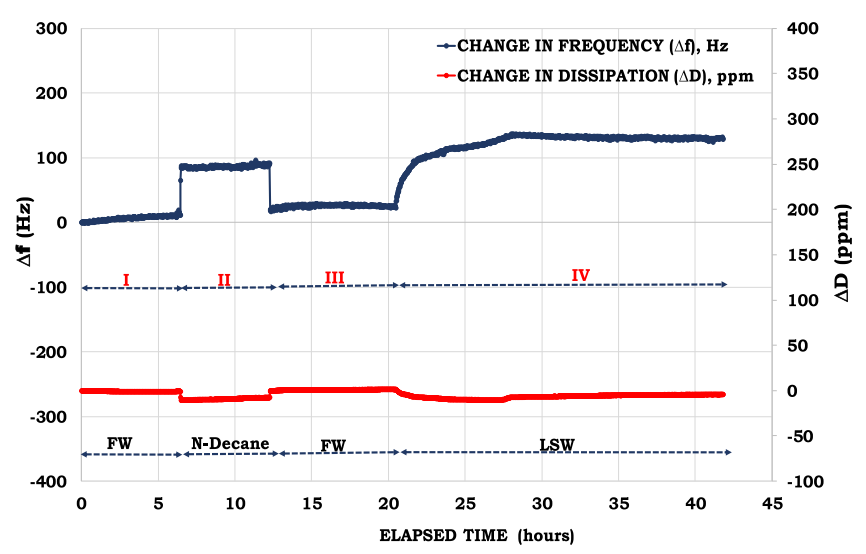

Fig. 20. Frequency and dissipation signals during FW/ $\mathrm{N}$-Decane/FW/LSW injection sequence using quartz sensor.

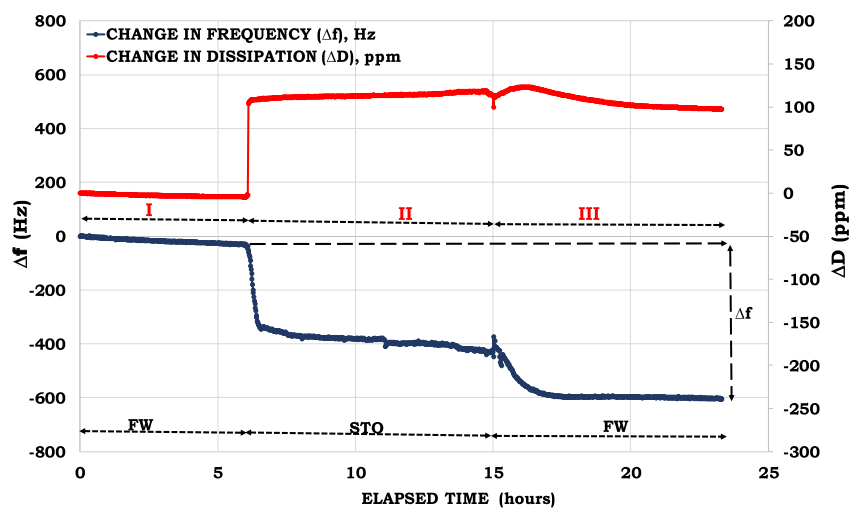

Fig. 21. Frequency and dissipation signals during FW/ $\mathrm{N}$-Decane/FW/LSW injection sequence using kaolinite sensor.

\subsection{Results with all the challenges resolved}

From Figures 19 and 20, it can be observed that the frequency and dissipation signals in an experiment with quartz sensor look relatively stable after the challenges were resolved. In addition, the $\mathrm{FW} / \mathrm{N}$-Decane/FW/LSW injection sequence depicted the lack of oil adsorption due to the negligible change in the FW frequency signal before and after the injection of $n$-decane in both cases (Part III of Fig. 19 and Part III of Fig. 20).

Considering the LSW injection stage (Part IV of Fig. 19 and Part IV of Fig. 20), it can be observed that the frequency signals were approximately the same $(90 \mathrm{~Hz}$ and $130 \mathrm{~Hz}$ respectively).

Figure 21 illustrates oil adhesion kinetic during $\mathrm{FW} / \mathrm{STO} / \mathrm{FW}$ injection sequence with kaolinite sensor. Some polar oil components from the STO were adsorbed onto the sensor surface. This was supported by the relatively large change in the FW frequency signal before and after injecting STO (Fig. 21) as compared to that of $n$-decane (Figs. 19 and 20).

\subsection{Potential applications of QCM-D}

The QCM-D can be employed in numerous surface interaction studies such as corrosion studies, dissolution and precipitation studies due to its high sensitivity and accuracy (Cattanach et al., 2011). In other words, it has the potential to screen possible source of impurities in the laboratory experimental set-up that might lead to erroneous results. This was shown by the corrosion challenges in the presented study. It can also be used for evaluating interactions on mineral surface such as Crude Oil/Brine/Rock (COBR) interactions. Hence, it has the capabilities to be used in wettability characterization studies as presented in this paper. This method has also the potential to screen possible brine compositions and chemicals that can alter the wettability, thereby accelerating production from oil reservoirs.

\section{Conclusion}

A fast and promising technique for surface investigations with the potential to be used in wettability characterization studies has been developed. The QCM-D technique combined with geochemical simulations can elucidate the underlying mechanisms in experimental data. In addition, the following conclusions are drawn base on the use of the development of the QCM-D technique in the study of oil adhesion onto mineral surfaces.

(I) The experimental set-up should be composed of highly corrosion resistant materials since the brine and the high temperature used in the experiment are suitable conditions for corrosion to take place.

(II) A pre-column with the designated minerals as the sensor coatings should be included in the experimental set-up so that the brine will be in equilibrium with the surface of the sensor thereby mitigating etching. Moreover, dissolution of minerals can also be investigated by the QCM-D technique.

(III) Brine and oil are immiscible and hence, displacement of one fluid with the other is not efficient due to the difference in the densities. During the experiment, the flow-cell was rotated in a way that maximizes 
the displacement of one fluid with the other. Hence, the flow-cell designed should be improved to allow efficient displacement of fluids.

(IV) To avoid the precipitation of salt, a constant temperature experimental set-up is needed.

Acknowledgments. The authors acknowledge the Research Council of Norway and the industry partners; ConocoPhillips Skandinavia AS, Aker BP ASA, Eni Norge AS, Total E\&P Norge $A S$, Equinor ASA, Neptune Energy Norge AS, Lundin Norway AS, Halliburton AS, Schlumberger Norge AS, Wintershall Norge AS and DEA Norge AS of The National IOR Centre of Norway for support.

\section{References}

Alagha L., Wang S., Yan L., Xu Z., Masliyah J. (2013) Probing adsorption of polyacrylamide-based polymers on anisotropic basal planes of kaolinite using quartz crystal microbalance, Langmuir 29, 12, 3989-3998.

Bluemmel J., Perschmann N., Aydin D., Drinjakovic J., Surrey T., Lopez-Garcia M., Kesslerc H., Spatz J.P. (2007) Protein repellent properties of covalently attached peg coatings on Nanostructured Sio2-based interfaces, Biomaterials 28, 32, 4739-4747.

Cao B., Stack A.G., Steefel C.I., DePaolo D.J., Lammers L.N., $\mathrm{Hu}$ Y. (2018) Investigating calcite growth rates using a quartz crystal microbalance with dissipation (QCM-D), Geochim. Cosmochim. Acta 222, 269-283.
Cattanach K., Ramachandran S., Jovancicevic V., Sherik A. (2011) A new methodology for monitoring corrosion under sales gas conditions using the quartz crystal microbalance, Paper presented at the CORROSION 2011.

Chandrasekaran N., Dimartino S., Fee C.J. (2013) Study of the adsorption of proteins on stainless steel surfaces using QCM-D, Chem. Eng. Res. Des. 91, 9, 1674-1683.

Dixon M.C. (2008) Quartz crystal microbalance with dissipation monitoring: enabling real-time characterization of biological materials and their interactions, J. Biomol. Techn. 19, 3, 151.

Ekholm P., Blomberg E., Claesson P., Auflem I.H., Sjöblom J., Kornfeldt A. (2002) A quartz crystal microbalance study of the adsorption of asphaltenes and resins onto a hydrophilic surface, J. Colloid Interface Sci. 247, 2, 342-350.

Erzuah S., Fjelde I., Omekeh A.V. (2018) Wettability estimation by oil adsorption using quartz crystal microbalance with disipation (QCM-D). Presented at EUROPEC, Copenhagen, 11-14 June.

Feiler A.A., Sahlholm A., Sandberg T., Caldwell K.D. (2007) Adsorption and viscoelastic properties of fractionated mucin (BSM) and bovine serum albumin (BSA) studied with quartz crystal microbalance (QCM-D), J. Colloid Interface Sci. 315, 2, 475-481.

Keller C.A., Kasemo B. (1998) Surface specific kinetics of lipid vesicle adsorption measured with a quartz crystal microbalance, Biophys. J. 75, 3, 1397-1402.

Nguyen T.H., Elimelech M. (2007) Adsorption of plasmid DNA to a natural organic matter-coated silica surface: kinetics, conformation, and reversibility, Langmuir 23, 6, 3273-3279. 